\title{
FEM-based Structural Optimization with Respect to Shakedown Constraints
}

\author{
Michael Heitzer \\ Central Institute for Applied Mathematics (ZAM), \\ Forschungszentrum Jülich, D-52425 Jülich, Germany \\ m.heitzer@fz-juelich.de \\ http://www.fz-juelich.de/zam/ZAMPeople/heitzer.html
}

\begin{abstract}
In this paper, a mathematical programming formulation is presented for the structural optimization with respect to the shakedown analysis of 3-D perfectly plastic structures on basis of a finite element discretization. A new direct algorithm using plastic sensitivities is employed in solving this optimization formulation. The numerical procedure has been applied to carry out the shakedown analysis of pipe junctions under multi-loading systems. The computational effort of the new approach is much lower compared to so called derivative-free search methods.
\end{abstract}

\section{Introduction}

In many technically meaningful problems of structures (e.g. in the plant manufacturing) under variable loads the nonlinear behaviour of the material must be considered. Inelastic analyses of the plastic (time-independent) or viscous (timedependent) behaviour are increasingly used to optimize industrial structures for safety and for an economic operation. Incremental analyses of the pathdependent plastic component behaviour are very time-consuming. The shakedown analysis belongs to the so-called direct or simplified methods which do not achieve the full details of plastic structural behaviour. The objective of shakedown analysis is the determination of an operation regime (i.e. safety margin, load carrying capacities) in which no early failure by plasticity effects has to be expected. Depending on the magnitude of loading, a structure can show the following structural failure modes:

- plastic collapse by unrestricted plastic flow at limit load,

- incremental collapse by accumulation of plastic strains over subsequent load cycles (ratchetting),

- plastic fatigue by alternating plasticity in few cycles, (low cycle fatigue),

- plastic instability of slender compression members (not considered here).

Within the Brite-EuRam Project LISA [17] a procedure is developed using the finite element discretization for direct calculation of the limit and shakedown load of structures made of ductile material. The shakedown analysis is formulated as optimization problem, such that it is easily reformulated for use in a structural 
optimization process. Determining optimal values for relevant design variables characterizing the geometrical shape as well as the material behaviour requires an efficient strategy to perform sensitivity analyses with respect to the design variables. Heyman [9] was the first to study the problem of optimal shakedown design. Recently, optimal plastic design of plates with holes under multi-loading systems [16] have been performed. The presented approach is suitable to general 3 -D structures which can be analyzed by a Finite Element code. In the new approach the sensitivity analysis is integrated in the formulation of the shakedown analysis. This permits an integrated treatment of structural and sensitivity analysis and results into easily applicable and efficient numerical algorithms. For a general review of sensitivity methods in nonlinear mechanics see [12]. Different types of problems may be considered in structural optimization:

- maximum shakedown load for a given structure (shape).

- optimum shape (e.g. minimum weight) for given shakedown load.

In this contribution we maximize the shakedown range of pipe junctions of variable thickness of the pipe and the junction.

\section{Concepts of Shakedown Analysis}

Static shakedown theorems are formulated in terms of stress and define safe structural states leading to an optimization problem for safe load domains. The maximum safe load domain is the load domain avoiding plastic failure (with the exception of plastic buckling). We restrict our presentation to perfectly plastic material and no elastic failure modes are considered (i.e. no elastic buckling or high cycle fatigue).

\subsection{Static or Lower Bound Shakedown Analysis}

The shakedown analysis starts from Melan's lower bound theorem for time variant loading for perfectly plastic material. It is assumed that the loads vary in a convex load domain $\mathcal{L}_{0}$ such that every load $P(t)=(\mathbf{b}(t), \mathbf{p}(t))$ which lays in $\mathcal{L}_{0}$ is generated by $N V$ non-degenerated load vertices $P_{j}$. The equilibrium conditions of the shakedown analysis and the yield criterion for the actual stresses have to be fulfilled at every instant of the load history. For the following considerations the VON MISEs function $F$ is preferred. The maximum enlargement of $\mathcal{L}_{0}$ is searched for which the structure is safe. The structure is safe against low cycle fatigue and against ratchetting if there is a stress field $\boldsymbol{\sigma}(\mathrm{t})$ such that the equilibrium equations are satisfied and the yield condition (with yield stress $\sigma_{y}$ ) is nowhere and at no instant $t$ violated.

$$
\begin{aligned}
\max & \alpha \\
\text { s.t. } & F(\boldsymbol{\sigma}(t)) \leq \sigma_{y} \text { in } V \\
& \operatorname{div} \boldsymbol{\sigma}(t)=-\alpha \mathbf{b}_{0}(t) \quad \text { in } V \\
& \boldsymbol{\sigma}(t) \mathbf{n}=\alpha \mathbf{p}_{0}(t) \quad \text { on } \partial V_{\sigma}
\end{aligned}
$$


for body forces $\alpha \mathbf{b}_{0}(t)$, surface loads $\alpha \mathbf{p}_{0}(t)$. By convexity of $\mathcal{L}_{0}$ the constraints need to be satisfied only in the load vertices $P_{j}$. This makes the problem time invariant for any deterministic or stochastic load history.

Problem (1) can be transformed into a finite optimization problem by FEM discretization. For structures with $N G$ Gaussian points in the FEM model one has to handle $O(N G)$ unknowns and $O(N G)$ constraints. The number of Gaussian points becomes huge for realistic discretizations of industrial structures (several 100000 points) and no effective solution algorithms for discretizations of the nonlinear optimization problem (1) are available. A method for handling such large-scale optimization problems for perfect plasticity is called basis reduction technique or subspace iteration [7], [18], [15]. This reduction technique generalizes the line search technique, well-known in optimization theory. Instead of searching the whole feasible region for the optimum a sequence of subspaces with a smaller dimension is chosen and one searches for the best value in these subspaces.

\section{Optimization Techniques}

Hooke and Jeeves coined the phrase direct search in a paper that appeared in 1961 [10]. It describes direct search by the sequential examination of trial solutions involving comparison of each trial solution with the best obtained up to that time together with a strategy for determining (as a function of earlier results) what the next trial solution will be. Many of the direct search methods are based on heuristics and recent analyses guarantee global convergence behavior analogous to the results known for globalized quasi-Newton techniques [14]. Direct search methods succeed because many of them can be shown to rely on techniques of classical analysis like bisection or golden section search algorithms. For simplicity, we restrict our attention here to unconstrained maximization of function $f: \mathbb{R}^{n} \rightarrow \mathbb{R}$. We assume that $f$ is continuously differentiable, but that information about the gradient of $f$ is either unavailable or unreliable. Because direct search methods neither compute nor approximate derivatives, they are often described as derivative-free. For a recent survey on direct search methods and genetic algorithms see [14] and [6], respectively. A classification of the most methods for numerical optimization can be done according to how many terms of the expansion they need [14], e.g.:

\section{- Newton's method (second order)} assumes the availability of first and second derivatives and uses the secondorder Taylor polynomial to construct local quadratic approximations of $f$.

- Steepest descent (first order) assumes the availability of first derivatives and uses the first-order Taylor polynomial to construct local linear approximations of $f$.

In this classification, zero-order methods do not require derivative information and do not construct approximations of $f$, such that they rely only on values of the objective function. For comparison with the proposed new method using 
plastic sensitivities the optimization codes PDS2 [14], SIMANN [5] and PIKAIA [1] are used.

\subsection{Quasi-Newton Methods}

A technique used for iteratively solving unconstrained optimization problems is the line search method. The method determines the optimal point on a given line (search direction). A back tracking algorithm is used which starts from an initial step length and decreases the step length until it is sufficient. The algorithm of Dennis/Schnabel is used to omit the exact solution of the one-dimension optimization on the search line [3].

The IMSL routines BCONF and BCONG are used for the maximization [11] if analytic gradients are available or not, respectively. BCONF/BCONG use a quasi-Newton method and an active set strategy to solve maximization problems subject to simple bounds $\mathbf{l}, \mathbf{u}$ on the variables. From a given starting point $\mathbf{x}^{c}$, an active set IA, which contains the indices of the variables at their bounds, is built. A variable is called a free variable if it is not in the active set. The routine then computes the search direction for the free variables from a positive definite approximation of the Hessian and the gradient evaluated at $\mathbf{x}^{c}$; both are computed with respect to the free variables. Routine BCONF calculates the gradient by a finite-difference method evaluated at $\mathbf{x}^{c}$. The search direction for the variables in IA is set to zero. A line search is used to find a better point $\mathbf{x}^{n}=\mathbf{x}^{c}+\lambda \mathbf{d}, \quad \lambda \in(0,1]$. Finally, the optimality conditions are checked for a suitable gradient tolerance. Another search direction is then computed to begin the next iteration. The active set is changed only when a free variable hits its bounds during an iteration or the optimality condition is met for the free variables but not for all variables in IA, the active set. In the latter case, a variable that violates the optimality condition will be dropped out of IA. For more details on the quasi-Newton method and line search, see [3].

The line search method needs for solving optimization problems a search direction $\mathbf{d}$. Here the search line is given by the gradients of the shakedown factor with respect to the design parameters. The shakedown factor is a solution of a nonlinear optimization problem and therefore the gradients are given by the sensitivities with respect to the design parameters. Using the chain rule the problem of the plastic structural behaviour can be reduced to the sensitivity analysis of the elastic structural response, which is a significant reduction of computational effort (see [8],[4]). The sensitivity analysis of the elastic response is performed by a finite-difference method for a small number of parameters, see [12] for alternative techniques.

\subsection{Pattern Search Method}

Pattern search methods are characterized by a series of exploratory moves that consider the behavior of the objective function at a pattern of points, all of which lie on a rational lattice. The exploratory moves consist of a systematic strategy for visiting the points in the lattice in the immediate vicinity of the 
current iterate [14]. For each move the parameter is varied and it is decided if there is an improvement, such that the procedure is a direct search. The used multi-directional search algorithm proceeds by reflecting a simplex through the centroid of one of the faces [14].

If one replaces a vertex by reflecting it through the centroid of the opposite face, then the result is also a simplex. The first single move is that of reflection which identifies the worst vertex in the simplex (i.e. the one with the least desirable objective value) and then reflects the worst simplex through the centroid of the opposite face. If the reflected vertex is still the worst vertex, then next choose the next worst vertex and repeat the process. The ultimate goals are either to replace the best vertex or to ascertain that the best vertex is a candidate for a maximizer. Until then, the algorithm keeps moving the simplex by flipping some vertex (other than the best vertex) through the centroid of the opposite face. An expansion step allows for a more progressive move by doubling the length of the step from the centroid to the reflection point, whereas a contraction steps allow for more conservative moves by halving the length of the step from the centroid to either the reflection point or the worst vertex. These steps allow a deformation of the shape of the original simplex.

\subsection{Simulated Annealing}

Simulated annealing is a global optimization method that distinguishes between different local maxima. Starting from an initial point, it randomly chooses a trial point within the step length of the user selected starting point. The function is evaluated at this trial point and its value is compared to its value at the initial point. When maximizing a function, any uphill step is accepted and the process repeats from this new point. An downhill step may be accepted, such that it can escape from local maxima. Downhill moves may be accepted; the decision is made by the Metropolis criteria. It uses $T$ (temperature) and the size of the downhill move in a probabilistic manner. The smaller $T$ and the size of the downhill move are, the more likely that move will be accepted. If the trial is accepted, the algorithm moves on from that point. If it is rejected, another point is chosen instead for a trial evaluation. As the optimization process proceeds, the length of the steps decline and the algorithm closes in on the global optimum. Since the algorithm makes very few assumptions regarding the function to be optimized, it is quite robust with respect to non-quadratic surfaces.

The simulated annealing algorithm of Corana et al. [2] was implemented and modified in [5] and is obtained from net NETLIB collection of mathematical software.

\subsection{Genetic Algorithms}

Genetic algorithms are a class of search techniques inspired from the biological process of evolution by means of natural selection. A top-level view of a canonical genetic algorithm could be as follows: Start by generating a set (population) of trial solutions, usually by choosing random values for all model parameters; then: 
1. Evaluate the (fitness) of each member of the current population.

2. Select pairs of solutions (parents) from the current population, with the probability of a given solution being selected made proportional to that solution's fitness.

3. Produce two new solutions (offspring) from the two solutions selected in (2).

4. Repeat steps (2)-(3) until the number of offspring produced equals the number of individuals in the current population.

5. Use the new population of offspring to replace the old population.

6. Repeat steps (1) through (5) until some termination criterion is satisfied.

The probability of a given solution being selected for breeding is proportional to the fitness, such that better trial solutions breed more often, the computational equivalent of natural selection. The production of new trial solutions from existing ones occurs through breeding. This involves encoding the parameters defining each solution as a string-like structure (chromosome), and performing genetically inspired operations of crossover and mutation to the pair of chromosomes encoding the two parents. The end result of these operations are two new chromosomes defining the two offsprings that incorporate information from both parents. No derivatives of the goodness of fit function with respect to model parameters need to be computed. In most real applications, the model will need to be evaluated a great number of times, such that the evaluation is computationally expensive.

The used PIKAIA subroutine[1] maximizes a user-supplied FORTRAN function using uniform one-point crossover, and uniform one-point mutation.

\section{Pipe-junction Subjected to Internal Pressure and Temperature Loads}

A pipe-junction subjected to internal pressure and temperature loads is analyzed as a simple example. The shakedown analyses are performed for perfectly plastic material with a yield stress $\sigma_{y}=250 \mathrm{~N} / \mathrm{mm}^{2}$. The inner diameters $D=39 \mathrm{~mm}$ and $d=15 \mathrm{~mm}$ of the pipe and of the junction are fixed, respectively. The length of the pipe and of the junction are $L=81.9 \mathrm{~mm}$ and $l=17.1 \mathrm{~mm}$ fixed, respectively. The variable dimensions are the wall-thickness $s$ and $t$ of the pipe and the junction, respectively. The meshes of the pipe-junction are generated my an automatic mesh-generator. The different models are discretized with 125 solid 20-node hexahedron elements (HEXEC20). The dimensions of the model are based on a pipe benchmark problem of PERMAS [15]. The FE-mesh and the essential dimensions of the different pipe-junctions are represented in Fig. 1. The pipe junction is subjected to two-parameter loading. Pressure $P$ and temperature difference $T$ vary independentally

$$
\begin{aligned}
& 0 \leq P \leq \alpha \mu_{1} P_{0}, \\
& 0 \leq T_{i} \leq \alpha \mu_{2} T_{0}, \quad 0 \leq \mu_{1}, \mu_{2} \leq 1 .
\end{aligned}
$$

$P_{0}$ and $T_{0}$ are a reference pressure and temperature difference, respectively. 

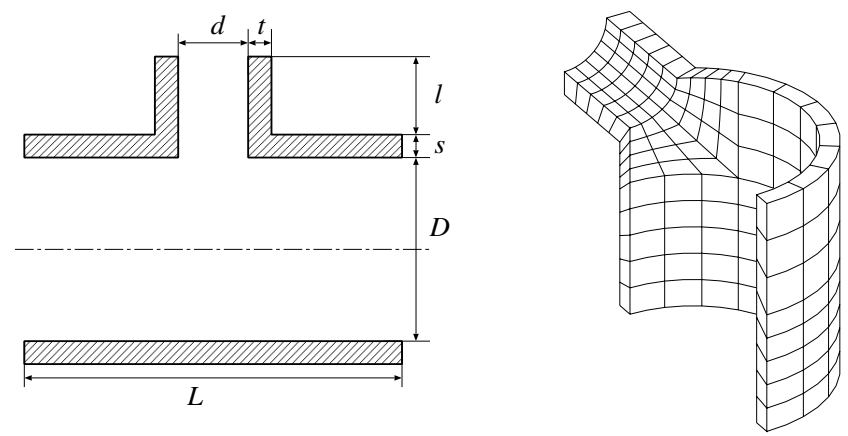

Fig. 1. FE-mesh and dimensions of the pipe-junction

The goal of the structural optimization in this example is to maximize the shakedown factor $\alpha_{s}$ for the wall-thickness $s$ and $t$ varying in given bounds:

$$
\begin{aligned}
\max & \alpha_{s} \\
\text { s.t. } & 0<s, t \leq 7.5 \\
\alpha_{s} & \text { solution of problem }(1)
\end{aligned}
$$

For pure pressure variation the optimal wall thickness of the pipe and of the junction will tend to infinity because of the decreasing elastic stresses. On the other hand for pure temperature variation the wall thickness of the pipe and of the junction will tend to zero. Therefore, different finite positive ratios between the initial pressure $P_{0}$ and the initial temperature $T_{0}$ are chosen. The design variables vary between bounds $0<s, t \leq 7.5 \mathrm{~mm}$ to guarantee that the chosen mesh of the pipe-junction is not degenerated, otherwise the chosen automatic mesh-generator leads to meshes with degenerated elements. The shakedown factor $\alpha_{s}$ is the solution of the corresponding shakedown optimization problem with the load domain $\mathcal{L}_{0}$ defined by $P_{0}$ and $T_{0}$.

Five different mathematical optimization codes using the FEM-based shakedown analysis are compared in the example. The direct methods PDS2, PIKAIA, SIMANN without the use of gradients and the IMSL routines BCONF and BCONG, using finite-difference method and analytical gradients, respectively. All codes use the same subroutine to calculate the shakedown load factor $\alpha_{s}$. The default parameters are used for all methods. PDS2 uses a fixed search pattern. BCONF performs a finite-difference method to estimate the gradient, such that for each gradient at least two additional shakedown analyses have to be performed. BCONG uses the given analytical gradients. The sensitivities are calculated using a finite-difference method for the elastic stresses and the algorithm described above.

A comparison of the different methods is shown in Table 1. In addition to the optimal values $s^{*}, t^{*}$ and the corresponding shakedown load magnitudes $P_{s}=\alpha_{s} P_{0}$ and $T_{s}=\alpha_{s} T_{0}$ the number of function calls (i.e. shakedown analyses) are given. The results for the methods are comparatively close. The values 
$s^{*}, t^{*}$ decrease for increasing temperatures as expected. The PDS2 algorithm is quite sensitive to the initial starting points, because of its fixed search scheme. Nevertheless with improved starting values the results for the pattern search method correspond well with the BCONG routine using the new implemented sensitivities. In allmost all cases the new method is the fastest method in terms of function calls. It is evident, that the use of the analytical gradients (BCONG) is preferable to the use of the finite-difference gradients (BCONF).

Table 1. Comparison of the different methods

\begin{tabular}{|c|c|c|c|c|c|c|}
\hline$T_{i}^{0} / P^{0}[\mathrm{~K} / \mathrm{MPa}]$ & Method & $\mathrm{s}^{*}[\mathrm{~mm}]$ & $t^{*}[\mathrm{~mm}]$ & $P_{s}[\mathrm{MPa}]$ & $T_{s}[\mathrm{~K}]$ & function calls \\
\hline \multirow{5}{*}{40} & PDS2 & 7.4665 & 6.8200 & 19.90 & 796 & 17 \\
\hline & GA & 6.3342 & 5.1185 & 20.72 & 829 & 17 \\
\hline & SIMANN & 5.5894 & 3.8753 & 19.56 & 782 & 59 \\
\hline & BCONF & 5.5654 & 3.7504 & 18.08 & 720 & 52 \\
\hline & BCONG & 7.5000 & 6.3041 & 19.75 & 790 & 12 \\
\hline \multirow{5}{*}{80} & PDS2 & 6.9828 & 6.6913 & 13.52 & 1082 & 50 \\
\hline & GA & 6.6958 & 5.4724 & 14.44 & 1155 & 10 \\
\hline & SIMANN & 5.9183 & 4.5799 & 13.56 & 1085 & 16 \\
\hline & BCONF & 4.1465 & 5.7304 & 11.65 & 932 & 45 \\
\hline & BCONG & 7.1101 & 4.9480 & 13.15 & 1052 & 4 \\
\hline \multirow{5}{*}{100} & PDS2 & 5.1344 & 4.0902 & 10.07 & 1007 & 22 \\
\hline & GA & 6.3168 & 4.5315 & 11.97 & 1197 & 13 \\
\hline & SIMANN & 6.8760 & 4.7475 & 12.21 & 1221 & 13 \\
\hline & BCONF & 4.5733 & 3.4209 & 10.71 & 1072 & 41 \\
\hline & $\mathrm{BCONG}$ & 4.1404 & 4.2517 & 10.10 & 1011 & 9 \\
\hline \multirow{5}{*}{120} & PDS2 & 6.9835 & 6.6906 & 10.35 & 1242 & 7 \\
\hline & GA & 7.3553 & 5.0235 & 10.47 & 1256 & 4 \\
\hline & SIMANN & 6.6813 & 5.0391 & 10.61 & 1273 & 19 \\
\hline & BCONF & 7.5000 & 4.4788 & 9.13 & 1096 & 27 \\
\hline & $\mathrm{BCONG}$ & 7.5000 & 6.7115 & 10.57 & 1268 & 6 \\
\hline \multirow{5}{*}{160} & PDS2 & 5.2488 & 4.6217 & 7.21 & 1154 & 41 \\
\hline & GA & 5.5639 & 5.0309 & 8.35 & 1336 & 11 \\
\hline & SIMANN & 4.4377 & 3.2308 & 7.50 & 1200 & 21 \\
\hline & BCONF & 4.9742 & 4.0640 & 7.81 & 1249 & 75 \\
\hline & BCONG & 3.8007 & 2.9375 & 6.96 & 1114 & 12 \\
\hline \multirow{5}{*}{200} & PDS2 & 6.0650 & 5.7721 & 6.60 & 1320 & 15 \\
\hline & GA & 5.3168 & 3.5315 & 6.26 & 1252 & 13 \\
\hline & SIMANN & 4.5928 & 3.7583 & 6.52 & 1304 & 12 \\
\hline & BCONF & 4.4038 & 3.9833 & 6.43 & 1286 & 68 \\
\hline & BCONG & 3.9576 & 3.5771 & 6.03 & 1207 & 5 \\
\hline
\end{tabular}

It has to be noticed that the function $f(s, t)=\alpha_{s}$ is not convex, such that probably local maxima exist in the region $0<s, t \leq 7.5 \mathrm{~mm}$. For instance in load level $T_{i}^{0} / P^{0}=100[\mathrm{~K} / \mathrm{MPa}]$ the resulting shakedown load magnitudes $P_{s}$ 
and $T_{s}$ are close for BCONG and PDS2, but for GA and SIMANN the values $s^{*}, t^{*}$ are fairly different. This may indicate that the global maximum in the region $0<s, t \leq 7.5 \mathrm{~mm}$ has not yet reached by BCONG and PDS2 but with the global methods GA and SIMANN. Additional computations suggest, that the temperature difference $T_{s} \approx 1400 \mathrm{~K}$ is the highest allowable temperature load in the region $0<s, t \leq 7.5 \mathrm{~mm}$.

\section{Conclusion}

Shakedown theorems are exact theories of classical plasticity for the direct computation of safety factors or of the load carrying capacity under varying loads. This method can be based on static and kinematic theorems for lower and upper bound analysis. Using Finite Element Methods more realistic modeling can be used for a more rational design. A mathematical programming formulation is presented for the structural optimization with respect to the shakedown analysis of 3-D perfectly plastic structures. A new direct algorithm using plastic sensitivities is employed in solving this optimization formulation. The numerical procedure has been applied to carry out the shakedown analysis of pipe junctions under multi-loading systems. The computational effort of the proposed method is lower compared to so-called derivative-free direct search methods.

\section{Acknowledgements}

Parts of this research have been funded by the Brite-EuRam III project LISA: FEM-Based Limit and Shakedown Analysis for Design and Integrity Assessment in European Industry (Project $\mathrm{N}^{\circ}$ : BE 97-4547, Contract $\mathrm{N}^{\circ}$ : BRPR-CT970595).

\section{References}

1. Charbonneau, P.: Genetic Algorithms in Astronomy and Astrophysics, The Astrophysical Journal (Supplements), 101 (1995) 309-334

2. Corana A., Marchesi M., Martini C., Ridella S.,: Minimizing Multimodal Functions of Continuous Variables with the "Simulated Annealing" Algorithm, ACM Transactions on Mathematical Software 13 (1987) 262-280

3. Dennis, J.E., Schnabel, R.B.: Numerical methods for unconstrained optimization, Engelwood Cliffs, N.J., Prentice Hall (1983)

4. Fiacco, A.: Introduction to Sensitivity and Stability Analysis in Nonlinear Programming, New York, Academic Press (1983)

5. Goffe, W.L., Ferrier,G., Rogers, J.: Global Optimization of Statistical Functions with Simulated Annealing, Journal of Econometrics 60 (1994) 65-99

6. Goldberg, D.E.: Genetic Algorithms in Search, Optimization, and Machine Learning Addison Wesley (1989)

7. Heitzer, M.: Traglast- und Einspielanalyse zur Bewertung der Sicherheit passiver Komponenten, Berichte des Forschungszentrums Jülich Jül-3704 (1999) 
8. Heitzer, M.: Structural optimization with FEM-based shakedown analyses Journal of Global Optimization (to appear).

9. Heyman, J.: Minimum weight of frames under shakedown loading. Journal of Engineering Mechanics, ASCE 84 (1958) 1-25

10. Hooke, R., Jeeves, T.A.: Direct search solution of numerical and statistical problems. J. Assoc. Comput. Mach. 8 (1961) 212-229

11. IMSL: Fortran Subroutines for Mathematical Application, Math/Library Vol. 1 \& 2 Visual Numerics, Inc. (1997)

12. Kleiber, M., Antúnez, H., Hien, T.D., Kowalczyk, P.: Parameter Sensitivity in Nonlinear Mechanics: Theory and Finite Element Computations, New York, J. Wiley \& Sons (1997)

13. König, J. A.: Shakedown of Elastic-Plastic Structures, Amsterdam and Warsaw, Elsevier and PWN (1987)

14. Lewis, R.M., Torczon, V., Trosset, M.W.: Direct search methods: then and now, Journal of Computational and Applied Mathematics 124 (2000) 191-207

15. PERMAS: User's Reference Manuals, Stuttgart, INTES Publications No. 202, 207, 208, 302, UM 404, UM 405 (1988)

16. Schwabe, F.: Einspieluntersuchungen von Verbundwerkstoffen mit periodischer Mikrostruktur, Phd-Thesis, RWTH-Aachen (2000)

17. Staat, M., Heitzer M.: LISA a European Project for FEM-based Limit and Shakedown Analysis, Nuclear Engineering and Design 206 (2001) 151-166

18. Stein, E., Zhang, G., and Mahnken, R.: Shakedown analysis for perfectly plastic and kinematic hardening materials, in Stein, E. (ed.): Progress in computational analysis of inelastic structures, Wien, Springer, 175-244 (1993) 